\title{
To Amputate or Not to Amputate: Management of latrogenic LASIK Flap Dehiscence and Epithelial Ingrowth with Overlying Pseudopterygium
}

\author{
Dean Ouano $^{a} \quad$ Rachel Huynh $^{b}$ Alyson Nicole Tukanc Nour Bundogjic \\ Majid Moshirfard, e, $f$ \\ aCoastal Eye Clinic, New Bern, NC, USA; bSchool of Medicine, University of Utah School \\ of Medicine, Salt Lake City, UT, USA; 'College of Medicine, University of Arizona College

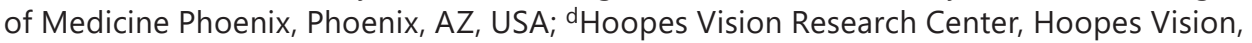 \\ Draper, UT, USA; eMoran Eye Center, University of Utah School of Medicine, Salt Lake City, \\ UT, USA; fUtah Lions Eye Bank, Murray, UT, USA
}

\section{Keywords}

Pseudopterygium · LASIK flap dehiscence · Conjunctival limbal autograft · Epithelial ingrowth · Conjunctivalization

\begin{abstract}
A 73-year-old male with a history of myopic laser-assisted in situ keratomileusis (LASIK) 20 years earlier presented with a late LASIK flap dehiscence, epithelial ingrowth, conjunctivalization, and the development of a pseudopterygium in the right eye. The findings were consistent with surgical trauma, likely occurring after corneal epithelial debridement to improve visualization during pars plana vitrectomy for retinal detachment repair 3 months earlier. The patient underwent epithelial ingrowth debridement, LASIK flap reapproximation and suturing, and a conjunctival limbal autograft from the contralateral eye. The surgery was completed successfully without the need for flap amputation. Postoperatively, the patient had an uneventful course with a well-healing conjunctival graft and no interface opacity or evidence of recurrent pseudopterygium of the right eye. The graft and corneal topography remained stable after subsequent cataract surgery.
\end{abstract}




\section{Case Reports in Ophthalmology}

\section{Introduction}

Traumatic flap dislocation after laser-assisted in situ keratomileusis (LASIK) has been documented as a postprocedural complication with the highest risk of occurrence in the immediate postoperative period (1-2\%) [1]. Dislocation months to years after surgery is less common [1-7], though there are reports up to 14 years postoperatively in cases of blunt ocular trauma $[5,8]$. Electron microscopy of a cornea 5 years after LASIK demonstrates incomplete wound healing, with disorganized collagen lamellae and primitive scar formation at the flap margin [9]. Therefore, LASIK flaps are susceptible to shearing forces even years after the completion of the procedure.

Flap disruption can lead to decreased visual acuity and haze $[4,10,11]$. In addition, ocular trauma can also result in the formation of a pseudopterygium, a nonprogressive fibrovascular adhesion due to growth factor activation in the setting of corneal epithelial damage [12]. We describe a patient who developed a pseudopterygium after LASIK flap dehiscence secondary to epithelial debridement in the setting of vitreoretinal surgery.

\section{Case Report}

A 73-year-old male with a history of myopic LASIK in 2001 presented for initial cataract evaluation. He was found to have LASIK flap dehiscence and epithelial ingrowth consistent with a pseudopterygium of the right eye due to suspected traumatic etiology. Three months before, he underwent vitreoretinal surgery in his right eye for phakic, macula-off retinal detachment (RD) involving pars plana vitrectomy (PPV), gas-fluid exchange, and scleral buckle. Corneal epithelial debridement was performed during the PPV to improve visualization, likely causing inadvertent inferior LASIK flap dehiscence. Subsequently, the flap was not repositioned, causing a prolonged postoperative epithelial defect that was noted upon referral for cataract evaluation.

Prior to RD and PPV, the patient had an uncorrected distance visual acuity of 20/30 OD and topography showing normal post-LASIK corneal changes (shown in Fig. 1a). Post-PPV topography taken at the time of initial cataract evaluation with Placido-ring videokeratography revealed significant distortion in the inferior one-half of the cornea with an induction of 6.89 diopters (D) of irregular astigmatism at axis $0^{\circ}$ (shown in Fig. 1b) with an uncorrected distance visual acuity of count fingers and a best-corrected visual acuity (BCVA) of 20/800. Anterior-segment optical coherence tomography (OCT) showed conjunctival ingrowth invading both the interface and superficial layer of the flap (shown in Fig. 1c). An extensive sheet of conjunctival tissue was found to be migrating from the inferior limbus to the interface (shown in Fig. 2a-c). Additionally, a 3+ nuclear cataract with dense fibrotic posterior subcapsular cataract (PSC) was seen. The patient was scheduled to undergo epithelial ingrowth debridement with LASIK flap repositioning and suturing and a conjunctival limbal autograft (CLAU) from the contralateral eye prior to cataract surgery.

\section{Operative Management}

The patient was prepared for surgery and draped in the usual sterile fashion after receiving peribulbar anesthesia. First, an incision was made at the perilimbal region of the inferior aspect of the right eye. Next, the pseudopterygium was carefully dissected off the cornea with removal of any residual necrotic tissue (shown in Fig. 3a-c). The underlying Tenon's capsule was removed down to the sclera. The conjunctiva was then excised from the flap interface. Due to the inward folding of the inferior flap edge and significant tissue loss, great care was taken to maximally preserve healthy tissue during excision. The traumatized LASIK flap was then cleaned with a lamellar dissector (shown in Fig. 3d-f), and the flap margin was

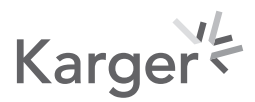



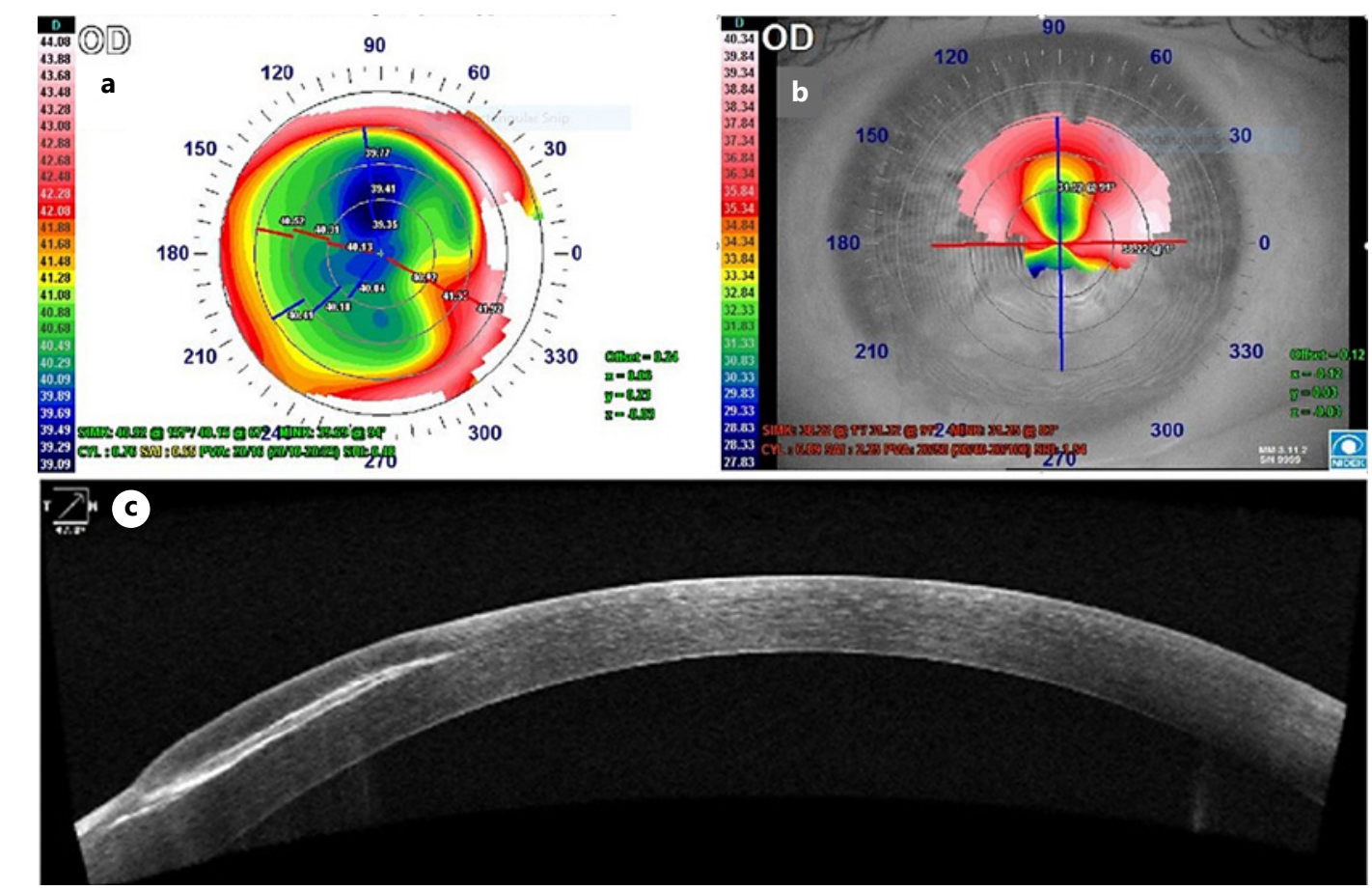

Fig. 1. Baseline topography of the right eye prior to PPV showing normal post-LASIK corneal changes (a); post-PPV topography taken at initial cataract evaluation showing loss of inferior curvature due to conjunctivalization (b); preoperative OCT with hyperreflective epithelium and conjunctival ingrowth invading both the interface and superficial layer of the LASIK flap (c). PPV, pars plana vitrectomy; LASIK, laser-assisted in situ keratomileusis; OCT, optical coherence tomography.

aligned to approximate its normal position (shown in Fig. 3g). Subsequently, the inferior margin of the LASIK flap was secured using 4 interrupted 10-0 nylon radial sutures (shown in Fig. 3h).

A prior peritomy complicated harvesting of a viable CLAU on the right eye to cover the residual exposed sclera. Thus, CLAU was harvested from the contralateral eye. First, a $6 \times 5-\mathrm{mm}$ graft dimension was identified at the inferior limbus of the left eye. It was marked with gentian violet, and the borders were trimmed using Wescott scissors and fine tying forceps (shown in Fig. 3i). A small volume of balanced saline solution was injected with a 30-gauge needle to hydrodissect the conjunctiva from Tenon's capsule, carefully considering making the graft as thin as possible (shown in Fig. 3j). The graft was hydrodissected anteriorly, extending just beyond the vascular arcades (shown in Fig. 3k). The anterior corners of the graft were tagged with 10-0 nylon sutures, leaving the left suture longer than the right, and the graft was removed entirely (shown in Fig. 3l, m). The CLAU was unrolled and placed at the inferior aspect of the flap on the recipient's right eye, using the 10-0 nylon tags for orientation. The graft was then sutured with 2 interrupted 10-0 nylon along the limbus and two 9-0 monofilaments at the posterior border (shown in Fig. 3n). Mitomycin C was avoided due to concerns for iatrogenic limbal stem cell dysfunction. After the procedure, a bandage contact lens was placed (shown in Fig. 3o).

\section{Postoperative Management}

Slit-lamp photos from postoperative day 1 through 2 months are shown in Figure $2 \mathrm{~d}$-i. The patient continued on a regimen of prednisolone QID OU and ofloxacin QID OU for 2 months postoperatively. All corneal sutures were removed by 5 weeks postoperatively. The patient's BCVA remained at 20/800 OD at all postoperative time periods. Postoperative topography revealed a residual $2.60 \mathrm{D}$ of astigmatism at axis $113^{\circ}$. At 2 months postoperatively, the graft 


\section{Case Reports in Ophthalmology}
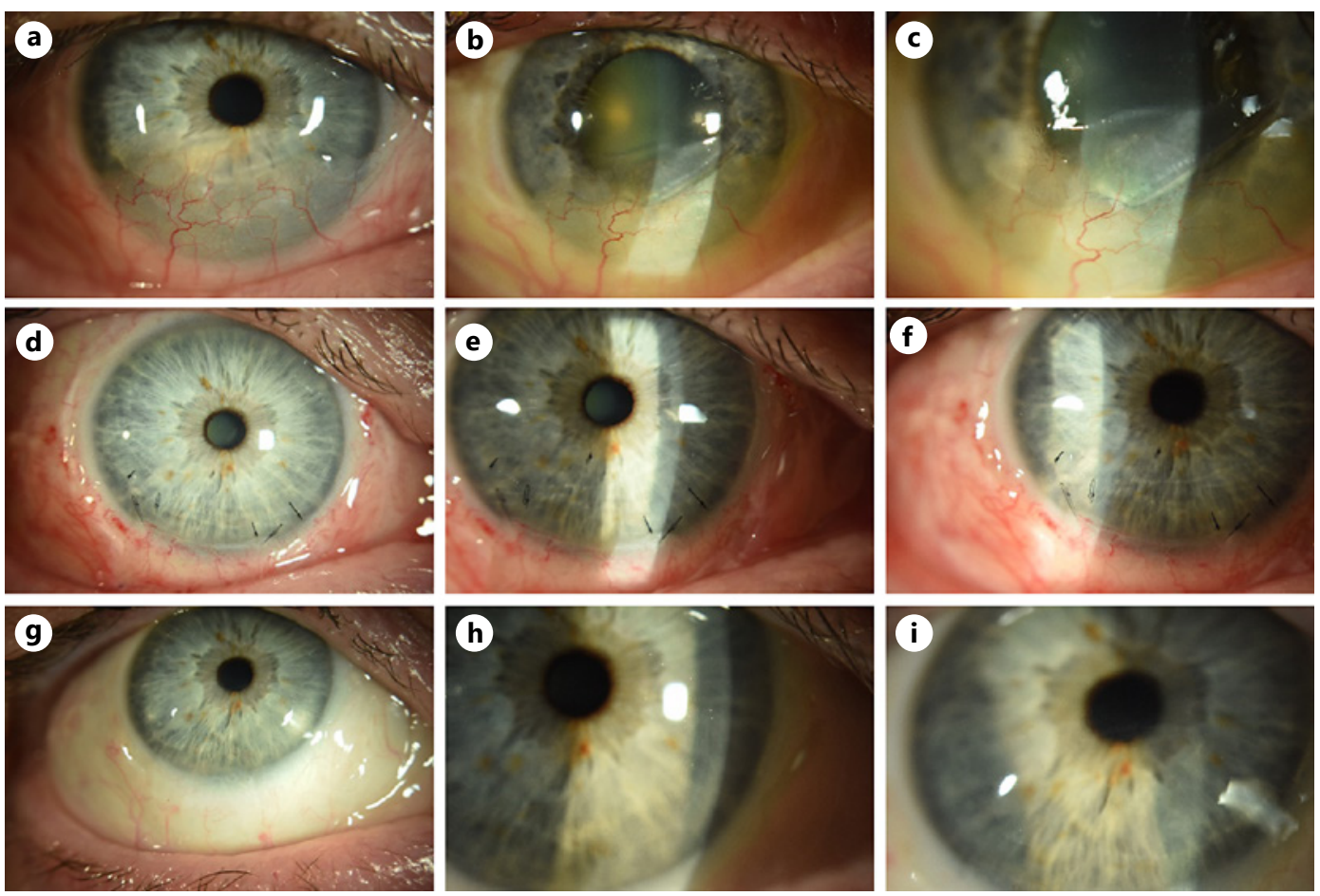

Fig. 2. Preoperative slit-lamp photos showing extensive conjunctival tissue migrating from the inferior limbus to over the LASIK flap and cornea (a-c); postoperative day 3 showing 6 sutures (d-f); postoperative month 2 showing removal of all sutures, complete healing of CLAU, and no re-epithelialization or fibrovascular scarring (g-i). LASIK, laser-assisted in situ keratomileusis; CLAU, conjunctival limbal autograft.

was healing well with no interface opacity or evidence of pseudopterygium recurrence in the right eye. The left eye showed normal epithelium with no signs of limbal stem cell deficiency (LSCD). Figure $4 \mathrm{a}$ and $\mathrm{b}$ demonstrate the improvement in the corneal surface regularity given the prior LASIK flap trauma and interface fibrosis.

After the ocular surface was stabilized, a cataract procedure was performed to remove the 3+ nuclear cataract with posterior subcapsular cataract in the right eye. A YAG laser capsulotomy was performed 1 month after cataract removal due to significant residual posterior capsule fibrosis. At 2 and a half months postoperatively, the corneal topography revealed $2.23 \mathrm{D}$ of astigmatism at axis $88^{\circ} \mathrm{OD}$, similar to the residual astigmatism after pseudopterygium removal. Figure $4 \mathrm{c}-\mathrm{e}$ demonstrate the improvement in corneal topography throughout his operative course. His final BCVA was 20/60 OD with a manifest refraction of $-0.75 \mathrm{D}$ of sphere and $-0.75 \mathrm{D}$ of cylinder at axis $137^{\circ}$. The limited final visual acuity was attributed to the partial thickness macular hole noted on macula OCT, though it is unclear whether this occurred due to his macula-off RD or post-PPV.

\section{Discussion}

Reports in the literature have demonstrated favorable visual outcomes after LASIK flap trauma when repaired in the acute setting [1]; however, management of subacute and chronic flap displacement is less characterized. This study presents a patient with prolonged traumatic LASIK flap dehiscence resulting in epithelial ingrowth and conjunctivalization of the right eye. Preservation of the LASIK flap was a primary goal. Despite initial concern for flap amputation due to substantial tissue damage, as shown by the extensive ingrowth visible on 


\section{Case Reports in Ophthalmology}
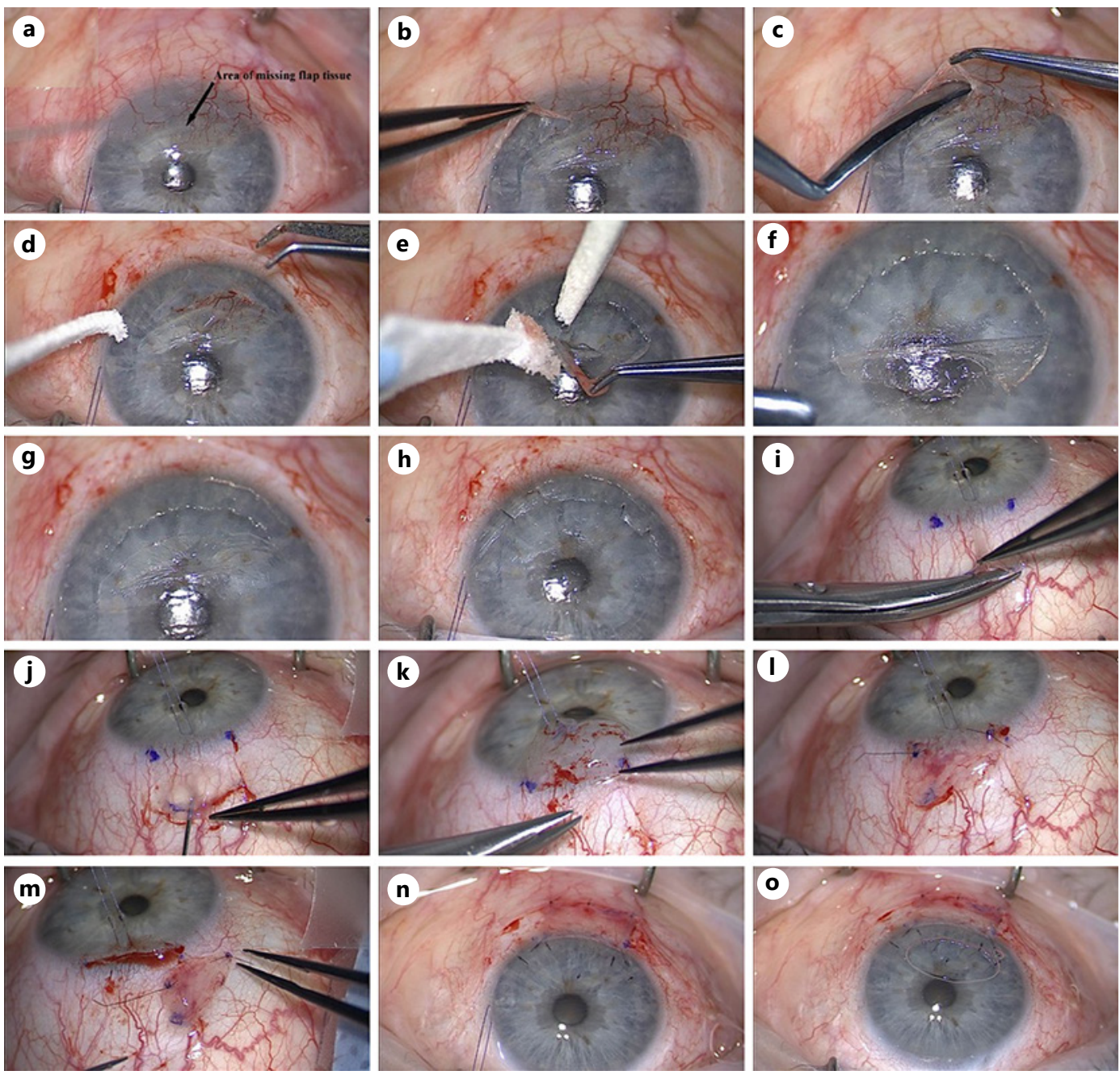

Fig. 3. Surgeon's view of the intraoperative steps. Removal of pseudopterygium in the right eye (a-c); debridement of the LASIK flap (d-f); suturing of the LASIK flap ( $\mathbf{g}-\mathbf{h})$; trimming, hydrodissection, and removal of CLAU in the left eye (i-m); CLAU sutured onto the right eye (n); BCL in place (o). LASIK, laser-assisted in situ keratomileusis; CLAU, conjunctival limbal autograft; BCL, bandage contact lens.

OCT, flap and stromal integrity were intact, thus amputation was avoided. Instead, meticulous debridement in conjunction with CLAU and suturing was effective in preserving native tissue. Salvaging the LASIK flap allowed for increased conservation of the normal corneal anatomy and reduced the risk of severe postoperative astigmatism. Further, the patient underwent cataract surgery, resulting in an improved BCVA from 20/800 to 20/60 OD with continued stability in his corneal topography and astigmatic correction.

Current approaches for preventing recurrent conjunctival epithelialization include mitomycin C, amniotic membrane grafts, and CLAU. Due to concerns for limbal stem cell dysfunction, mitomycin C was not used intraoperatively. CLAU was instead chosen to optimize corneal healing and prevent further conjunctivalization of the affected eye. CLAU graft size was considered in operative planning, as harvesting a large piece of limbal tissue could induce iatrogenic LSCD at the donor site [13]. This patient shows no signs of LSCD to date, which would be observed as a loss of limbal palisades of Vogt, stippling of corneal epithelium, and recurrent erosions [14]. 


\section{Case Reports in Ophthalmology}

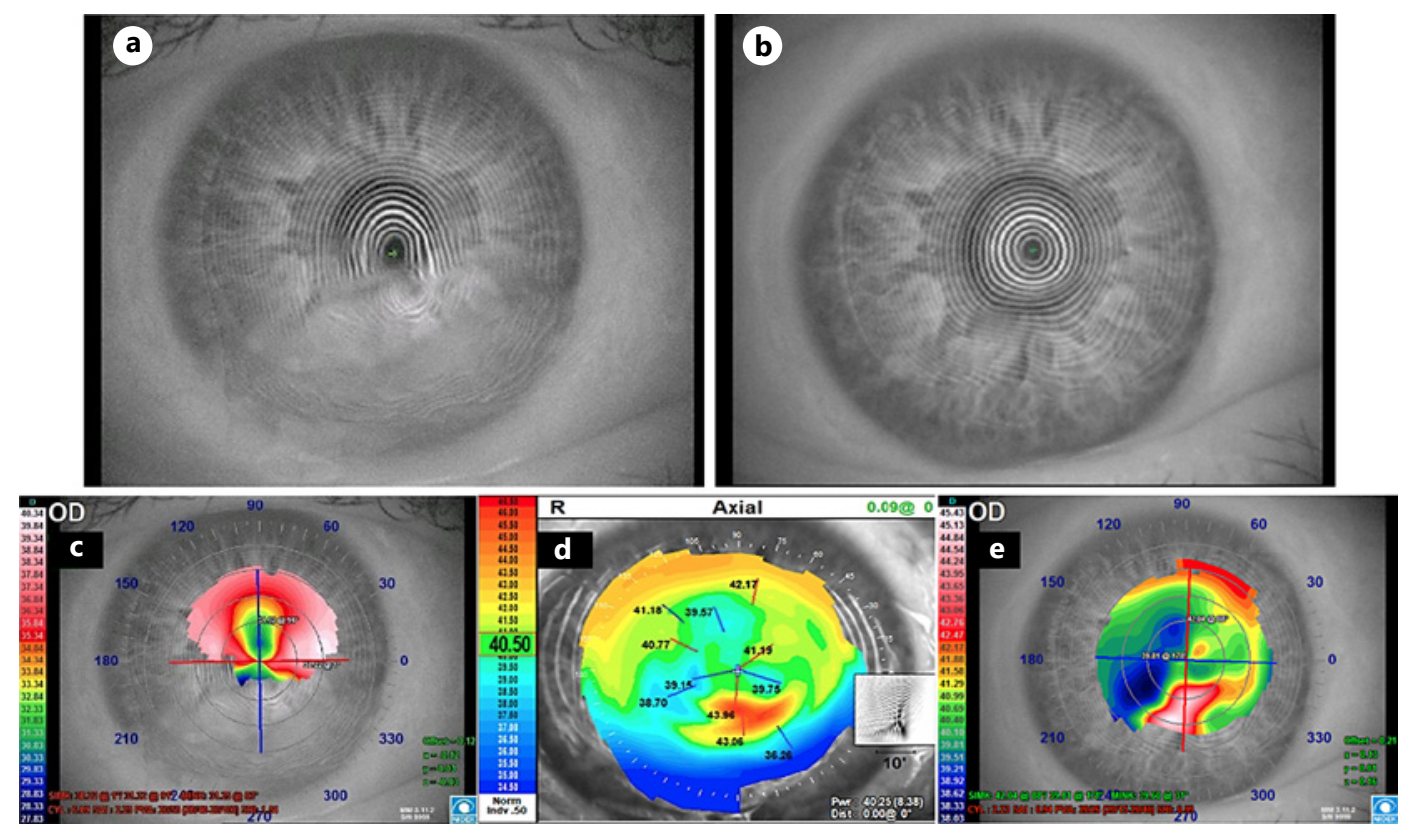

Fig. 4. Preoperative Placido disc with distorted inferior mires demonstrating irregular astigmatism (a); postoperative Placido disc with partial restoration of regularity at inferior mires demonstrating improved astigmatism (b); corneal topography after PPV with LASIK flap dehiscence and pseudopterygium development (c); corneal topography 3 months after pseudopterygium removal (d); corneal topography 6 months after pseudopterygium removal and 2 months after cataract removal (e). PPV, pars plana vitrectomy; LASIK, laser-assisted in situ keratomileusis.

\section{Conclusions}

We report the management of pseudopterygium removal, LASIK flap preservation and suturing, and conjunctival grafting in the setting of a prolonged epithelial defect. The extensive photography highlighted in this case allows for full appreciation of the patient's pathology and intraoperative flap revision with CLAU grafting. This case underscores the importance of recognizing iatrogenic LASIK flap damage. Specifically, retinal surgeons performing epithelial debridement for better visualization need to be conscientious of the presence of a LASIK flap. Thus, a thorough surgical history should be obtained, especially in myopic patients, to reduce the risk of iatrogenic LASIK flap dehiscence.

\section{Acknowledgments}

Special thanks are due to Shannon McCabe, MD, and Yasmyne Ronquillo, MD, for their assistance with editing of this manuscript.

\section{Statement of Ethics}

This study protocol was reviewed and approved by the Hoopes Vision Ethics Committee on July 22, 2021. Written informed consent was obtained from the patient concerning the disclosure and use of medical information regarding the medical case for publication purposes, including publication of the accompanying images. 


\section{Conflict of Interest Statement}

The authors have no conflicts of interest to declare.

\section{Funding Sources}

This study was funded by an unrestricted grant from Research to Prevent Blindness (RPB), 360 Lexington Avenue, twenty-second Floor New York, NY, USA. No support was received for the publication of this article.

\section{Author Contributions}

All authors take responsibility for the integrity of the work and have given final approval to the version to be published. Dean Ouano was the operating surgeon in the case and provided the images used for this case report, detailed the operative management involved in this case, and revised the manuscript. Rachel Huynh, Alyson Nicole Tukan, Nour Bundogji, and Majid Moshirfar contributed to the writing and revising of the manuscript. All authors and those acknowledged have agreed to submit to the current journal, gave final approval of the version to be published, and agree to be accountable for all aspects of the work.

\section{Data Availability Statement}

All data generated or analyzed during this study are included in this article. Further enquiries can be directed to the corresponding author.

\section{References}

1 Tsai TH, Peng KL, Lin CJ. Traumatic corneal flap displacement after laser in situ keratomileusis (LASIK). Int Med Case Rep J. 2017 Apr 19;10:143-8. Available from: https://pubmed.ncbi.nlm.nih.gov/28458585/.

2 Galvis V, Tello A, Ortiz AI, Quintero MP, Parra MM, Blanco NA. Traumatic corneal flap avulsion and loss 13 years after LASIK. Saudi J Ophthalmol. 2019 Apr-Jun;33(2):172-6.

3 Kim JS, Chung B, Lee T, Kim WC, Kim TI, Kim EK. Management of long-standing partially torn and flipped laser in situ keratomileusis flaps. J Cataract Refract Surg. 2015 Feb;41(2):464-7.

4 Shih LY, Peng KL, Chen JL. Traumatic displacement of laser in situ keratomileusis flaps: an integrated clinical case presentation. BMC Ophthalmol. 2021 Apr 13;21(1):177.

5 Ting DSJ, Danjoux J-P. Late-onset traumatic dislocation of laser in situ keratomileusis corneal flaps: a case series with many clinical lessons. Int Ophthalmol. 2019 Jun;39(6):1397-403.

6 Xiao J, Jiang C, Zhang M, Jiang H, Li S, Zhang Y. When case report became case series: 45 cases of late traumatic flap complications after laser-assisted in situ keratomileusis and review of Chinese literature. Br J Ophthalmol. 2014 Sep;98(9):1282-6.

7 Iskander NG, Peters NT, Anderson Penno E, Gimbel HV. Late traumatic flap dislocation after laser in situ keratomileusis. J Cataract Refract Surg. 2001 Jul;27(7):1111-4.

8 Holt DG, Sikder S, Mifflin MD. Surgical management of traumatic LASIK flap dislocation with macrostriae and epithelial ingrowth 14 years postoperatively. J Cataract Refract Surg. 2012 Feb;38(2):357-61.

9 Abahussin M, Hayes S, Edelhauser H, Dawson DG, Meek KM. A microscopy study of the structural features of post-LASIK human corneas. PLoS One. 2013 May 1;8(5):e63268.

10 Sridhar MS, Rapuano CJ, Cohen EJ. Accidental self-removal of a flap--a rare complication of laser in situ keratomileusis surgery. Am J Ophthalmol. 2001 Nov;132(5):780-2.

11 Haw WW, Manche EE. Successful corneal flap replacement following complete traumatic flap amputation after laser-assisted in situ keratomileusis. Arch Ophthalmol. 2004 Feb;122(2):275-6.

12 Pterygium - StatPearls - NCBI Bookshelf [Internet]. [cited 2021 Jun 4]. Available from: https://www.ncbi.nlm. nih.gov/books/NBK558907/. 


\section{Case Reports in Ophthalmology}

13 Saghizadeh M, Kramerov AA, Svendsen CN, Ljubimov AV. Concise review: stem cells for corneal wound healing. Stem Cells. 2017 Oct;35(10):2105-14.

14 Diagnosis and management of limbal stem cell deficiency - American academy of ophthalmology [Internet]. [cited 2021 May 25]. Available from: https://www.aao.org/eyenet/article/diagnosis-management-of-limbalstem-cell-deficienc. 